\title{
Ultraprecise measurement of resonance shift for sensing applications
}

\author{
Ciyuan Qiu, Jianbo Chen, and Qianfan Xu* \\ Department of Electrical and Computer Engineering, Rice University, Houston, Texas 77005, USA \\ *Corresponding author: qianfan@rice.edu
}

Received October 3, 2012; revised November 8, 2012; accepted November 8, 2012;

posted November 8, 2012 (Doc. ID 177426); published November 30, 2012

\begin{abstract}
Resonator-based optical sensors detect the change of refractive index in the environment by measuring the resonance shift. The sensitivity of such sensors is determined by how precise one can locate the resonant wavelength, which is thought to be limited by the bandwidth and the quality factor of the resonator. Here we show that, with a tunable resonator, one can determine the resonant wavelength with ultrahigh precision. Using a silicon microring resonator with an embedded $\mathrm{p}-\mathrm{i}-\mathrm{n}$ junction for electro-optic tuning, whose quality factor is only 14,000, we measured the resonant wavelength with a resolution of $0.06 \mathrm{pm}$, which corresponds to an index sensitivity of $\sim 10^{-7}$. This resonance measurement for sensing purposes can be done using a fixed-wavelength laser. (c) 2012 Optical Society of America
\end{abstract}

OCIS codes: $230.3120,130.6010$.

Biosensors with single-molecule sensitivity have been demonstrated using dielectric resonators with ultrahigh quality factor $(Q)[\underline{1}, 2]$ or plasmonic resonators with ultrasmall mode volume [3]. These sensors detect the change in the refractive index of the material surrounding the resonator by measuring the resonance shift of the resonator. While silicon photonics provides a vast capability for low-cost and large-scale optoelectronic integration and forms a promising platform for optical sensing, relatively limited $Q$ of microresonators based on silicon (typically $10^{4}-10^{6}$ ) has limited their sensitivity $[\underline{4}, \underline{5}]$. Similar constraints apply to other material platforms such as high-index-doped silica [6,7]. Although the sensitivity can be greatly improved by a resonance peak fitting algorithm [8], the precision is still constrained by such adverse effects as spectra ripples due to reflections in the optical path, fluctuation of the laser power, and fluctuation of fiber-to-chip coupling efficiency. These adverse effects can distort the measured spectra and makes a precise fitting difficult.

The transmission spectrum of a resonator $T(\lambda)$ typically has a Lorentzian shape, and the slope of the spectrum is zero at the resonant wavelength $\lambda_{R}$ [i.e., $T^{\prime}\left(\lambda_{R}\right)=0$ ], which reduces the accuracy of an absolute wavelength measurement. Here we precisely measure the resonance by finding the zero-slope point of the spectrum. To do that, the resonance is tuned periodically with a frequency $f$ so that

$$
\lambda_{R}(t)=\lambda_{0}+\delta \lambda \cdot \cos (2 \pi f t),
$$

where $\lambda_{0}$ is the central wavelength and $\delta \lambda$ is the range of resonance shift. When a CW laser at wavelength $\lambda$ is sent through the resonator, optical output power will have a fundamental frequency component $E(f)$ and a secondharmonic component $E(2 f)$, which satisfy

$$
\begin{gathered}
E(f) \propto P_{\text {in }} \cdot \delta \lambda \cdot T^{\prime \prime}(\lambda), \\
E(2 f) \propto P_{\text {in }} \cdot \delta \lambda^{2} \cdot T^{\prime \prime}(\lambda),
\end{gathered}
$$

where $P_{\text {in }}$ is the input optical power. When the laser wavelength is aligned exactly with the central wavelength $\lambda_{0}$, the $f$ component vanishes from the modulated optical output while the second-harmonic component is maximized [see the inset of Fig. 2(a) below]. In this way, we can use the amplitude ratio $R=$ $|E(2 f) / E(f)|$, which peaks sharply when $\lambda=\lambda_{0}$, to precisely locate $\lambda_{0}$. When the optical power drifts due to factors other than the resonator, such as fluctuations in laser power and coupling efficiency, both $E(2 f)$ and $E(f)$ changes in same way, while their ratio $R$ does not change. The insensitivity to power fluctuations is a significant advantage when the sensor is used to monitor slow refractive index changes in a biological environment.

As proof of concept, here we demonstrate a sensor based on a silicon microring modulator [9-13], which is an electro-optic tunable microring resonator with an embedded p-i-n junction (see the inset of Fig. 1). This device has small size [9], low power consumption [11], and the capability of large-scale integration [12]. We demonstrate a wavelength resolution $<10^{-3} \cdot \bar{\Delta} \lambda_{\mathrm{FWHM}}$, which translates to a sensitivity to effective index variation of

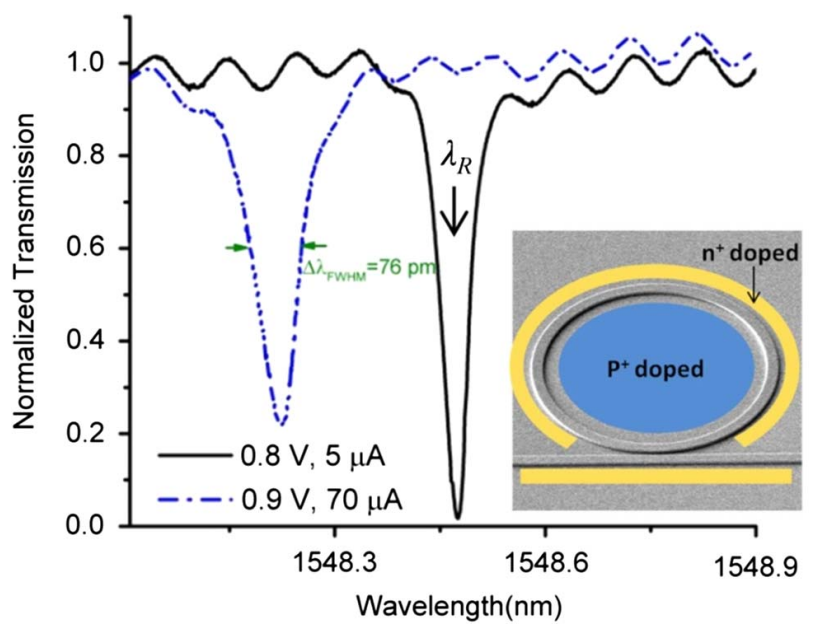

Fig. 1. (Color online) Transmission spectra of a silicon microring modulator at the bias voltages of $0.8 \mathrm{~V}$ (black solid) and $0.9 \mathrm{~V}$ (blue dotted-dashed). Inset, scanning electron microscopy image of the microring modulator after etching. The false color shows the implanted areas. 
the order of $10^{-7}$ [4]. The demonstrated high sensitivity is limited by the instruments, while the fundamental limit of wavelength resolution can be estimated to be $\sim 10^{-7} \cdot \Delta \lambda_{\text {FWHM }}$. Moreover, the measurement can be done with a fixed-wavelength laser instead of a tunable laser, which significantly reduces the complicity and cost of a sensor system.

The silicon microring modulator is fabricated in a complementary metal-oxide semiconductor photonics foundry at the Institute of Microelectronics of Singapore. The fabrication starts on a silicon-on-insulator wafer with a $220 \mathrm{~nm}$ thick top silicon layer and a $3 \mu \mathrm{m}$ thick buried oxide layer. Devices are formed by rib waveguides with widths of $500 \mathrm{~nm}$ on a $50 \mathrm{~nm}$ slab, which supports only the fundamental TE mode. The microring resonator with a diameter of $10 \mu \mathrm{m}$ is side-coupled to a straight waveguide as shown in the inset of Fig. 1 . The slab regions in and out of the ring are doped to $p^{+}$and $n^{+}$types, respectively, to form a $p-i-n$ junction across the ring. Inverse tapers with $180 \mathrm{~nm}$ wide tips are used to couple light into the waveguides.

The transmission spectrum at a bias voltage of $0.8 \mathrm{~V}$ is shown as the black solid curve in Fig. 1. At the resonant wavelength $\lambda_{R}$, the destructive interference between the light traveling through the ring resonator and the light traveling through the waveguide causes a dip in the transmission spectrum at resonance. The optical energy is lost in the resonator due to bending loss, sidewall scattering and free-carrier absorption. The rate of these losses and the strength of the coupling between the ring and the waveguide determine the $Q$ of the resonance [4]. When more free carriers are injected into the $\mathrm{p}$-i-n junction with a forward bias voltage of $0.9 \mathrm{~V}$, the resonant wavelength is blueshifted. The depth of the resonance decreases due to the free-carrier absorption effect [14]. At this bias voltage, the measured FWHM of the spectrum is $\Delta \lambda_{\mathrm{FWHM}}=76 \mathrm{pm}$, corresponding to a $Q$ of 20,000 . In theory, by fitting the spectrum, the resonant wavelength can be determined with a resolution much smaller than $\Delta \lambda_{\text {FWHM }}[3,8]$. However, the measured spectra often contain complicated features caused by structures away from the resonator. One can see ripples in the offresonance region, which is due to a weak Fabry-Perot cavity formed from the residue reflections at the ends of the waveguides. These ripples overlap with the resonance notch and make it slightly unsymmetrical. This type of distortion affects the accuracy of spectra fitting, especially when the extinction ratio (ER) of the resonance is relatively low.

The approach we demonstrate here is based on periodically tuning the resonator. The spectral features that are not originated from the resonator are not affected by the tuning and will thus not affect the precision of the measurement. In this demonstration, the low- $Q$ resonance of the silicon microring modulator biased at $0.9 \mathrm{~V}$ is tuned at a repetition frequency of $f=10 \mathrm{kHz}$ [10]. The amplitude of the tuning signal is set to be $4 \mathrm{mV}_{\text {rms }}$, which is the minimal output of the source.

The harmonic ratios $R$ at different input wavelengths are obtained with a lock-in amplifier and are shown as the red dots and lines in Fig. 2(a). The $R$ spectrum has a peak much sharper than the notch in the transmission spectrum. The zoom-in spectra in Fig. 2(b) shows more
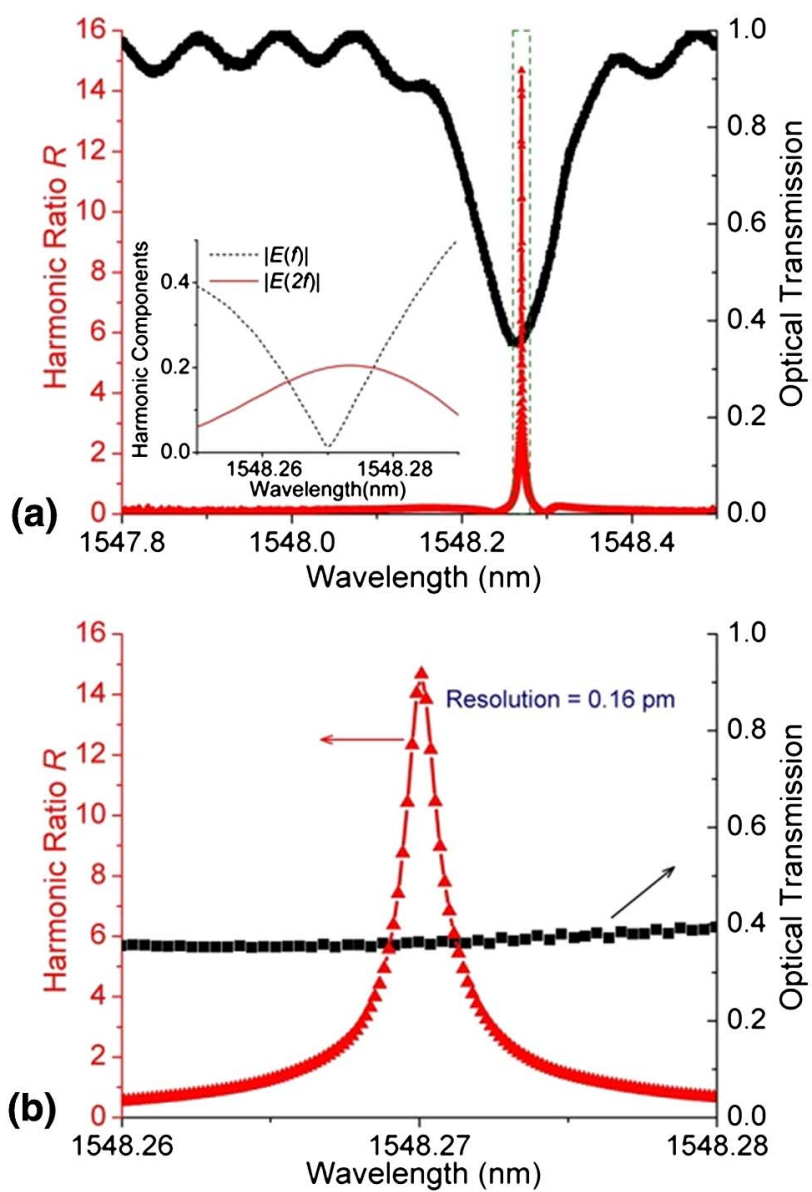

Fig. 2. (Color online) Spectra of normalized optical transmission (black squares) and harmonic ratio $R$ (red triangles) of the silicon microring modulator. (a) Broad spectra over a $0.7 \mathrm{~nm}$ range. The inset shows the harmonic components around the resonance wavelength (b) zoom-in spectra over a 20 pm wavelength range shown as the dashed rectangle in (a).

clearly the contrast between the sharpness of the transmission spectrum and $R$ spectrum. In this narrow wavelength range, the transmission spectrum is essentially flat, while the sharp peak in the $R$ spectrum marks the central wavelength with a fine resolution of $0.16 \mathrm{pm}$, which is limited by the tuning step size of the laser since the external-cavity laser used in the experiment is tuned with a step motor. Note that both the ER and $Q$ of the resonance measured from the average output power will decrease a little due to periodically tuning. However, that does not affect the wavelength resolution.

A refractive index sensor made of this tunable resonator can be probed with a fixed-wavelength laser instead of a tunable laser. In this regard, we fix the laser wavelength while tuning the central resonant wavelength $\lambda_{0}$ with a DC bias. The resonance shift depends on the DC current through the $p$-i-n junction. The amplitude of the $10 \mathrm{kHz}$ AC tuning signal is set to be $0.55 \mathrm{~V}_{\mathrm{rms}}$. The DC bias scan step is $0.1 \mathrm{~V}$ and $2 \mathrm{mV}$ for the coarse and fine scan shown in Figs. 3(a) and 3(b), respectively. In this demonstration, an overcoupled resonator device with an ER of $8.4 \mathrm{~dB}$ and a $Q$ of 14,000 is used. Figure $3(\mathrm{a})$ shows the measured optical transmission and harmonic ratio $R$ versus the bias current. The resonant wavelength shifts 
(a)

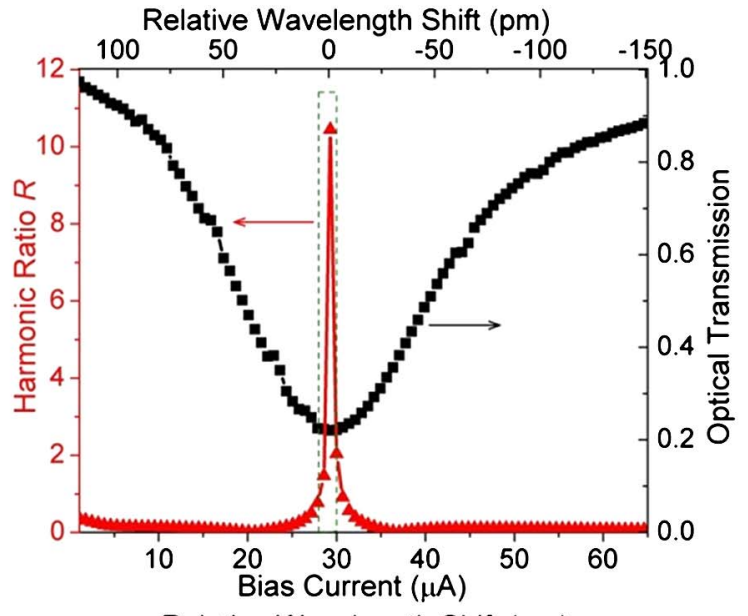

(b)

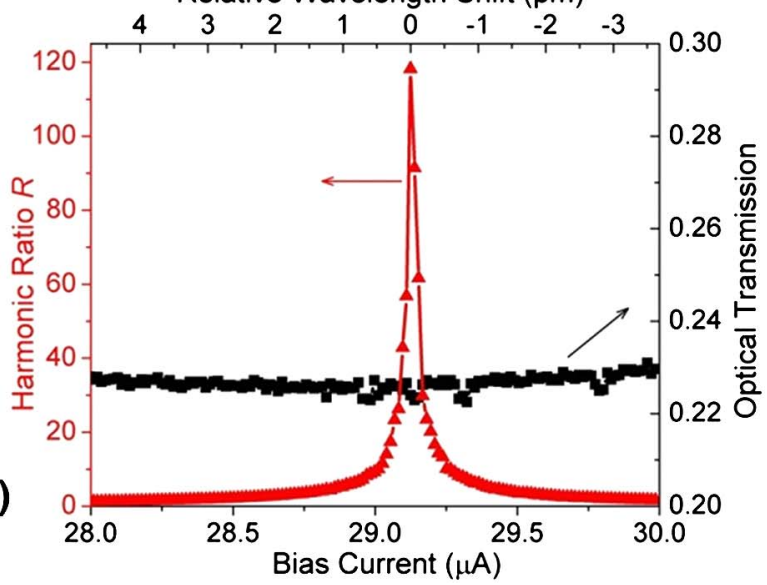

Fig. 3. (Color online) (a) Normalized optical transmission (black squares) and the harmonic ratio $R$ (red triangles) versus the DC bias current applied on the microring modulator. The top axis shows the resonant wavelength shift corresponding to the bias current. (b) High-resolution ( $\sim 0.06 \mathrm{pm})$ measurements within a narrow bias current range $(\sim 2 \mu \mathrm{A})$ inside the dashed rectangle in (a).

linearly with respect to the bias current at a rate of $4.1 \mathrm{pm} / \mu \mathrm{A}$. Again, one can see $R$ peaks sharply when the resonance aligns exactly with the laser wavelength. When zoomed in to a $2 \mu \mathrm{A}$ tuning range, one can still see the sharp peak with a resolution of $0.06 \mathrm{pm}$, which equals $5.3 \times 10^{-4} \bullet \Delta \lambda_{\text {FWHM }}$ and $3.9 \times 10^{-8} \bullet \lambda_{0}$. This resolution is now limited by the resolution of the DC bias source. Since the well-resolved wavelength shift of $0.06 \mathrm{pm}$ comes from an effective index change of $1.6 \times 10^{-7}$, a biosensor built on this technology can have a correspondingly high sensitivity, which is comparable to what is observed in the single-molecule sensing experiment [1] . In comparison, the optical transmission changes less than $1 \%$ in the entire tuning range in this figure. The noises on the transmission spectrum are from the fluctuations in chip-to-fiber coupling and in laser power.

The demonstrated resolution is limited by the step sizes of the tunable laser and the DC bias source, both of which can be improved by using better instruments.
The ultimate limit of the wavelength resolution through this approach will come from the relative intensity noise (RIN) level of the laser, which translates to a noise floor for $E(f)$ measurements. To be able to locate the resonant wavelength $\lambda_{0}$ within a range of $2 \varepsilon$, the modulation signal $|E(f)|$ at $\lambda_{0} \pm \varepsilon$ needs to be above the noise level, while $|E(f)|$ at $\lambda_{0}$ should be below the noise level (and thus be judged as zero). Using a laser with a typical RIN of $-140 \mathrm{~dB} / \mathrm{Hz}$, and with a $1 \mathrm{~Hz}$ bandwidth on the lock-in amplifier, the resolution limit is of the order of $10^{-7} \bullet \Delta \lambda_{\text {FWHM }}$, which corresponds to an effective index sensitivity of the order of $10^{-11}$. Note that the accuracy of the measurement will also be affected by the drift in laser wavelength and the bias voltage. Highly stable sources are required to realize the ultrahigh sensitivity.

In conclusion, we demonstrate a method to build ultrasensitive sensors for the accurate evaluation of the change in the refractive index. The demonstration is based on an electro-optic tunable optical resonator on silicon with a moderate $Q$. This method is immune to fluctuation in laser power and coupling. This approach will help the highly integrated optical sensors built on silicon to achieve single-molecule sensitivity.

This work was partially supported by Air Force Office of Scientific Research (AFOSR) (grants FA9550-12-10261).

\section{References}

1. A. M. Armani, R. P. Kulkarni, S. E. Fraser, R. C. Flagan, and K. J. Vahala, Science 317, 783 (2007).

2. L. He, Ş. Özdemir, J. Zhu, W. Kim, and L. Yang, Nature Nanotechnol. 6, 428 (2011).

3. I. Ament, J. Prasad, A. Henkel, S. Schmachtel, and C. Sönnichsen, Nano Lett. 12, 1092 (2012).

4. D. X. Xu, A. Densmore, A. Delâge, P. Waldron, R. McKinnon, S. Janz, J. Lapointe, G. Lopinski, T. Mischki, E. Post, P. Cheben, and J. H. Schmid, Opt. Express 16, 15137 (2008).

5. K. De Vos, I. Bartolozzi, E. Schacht, P. Bienstman, and R. Baets, Opt. Express 15, 7610 (2007).

6. D. Duchesne, M. Peccianti, M. R. E. Lamont, M. Ferrera, L. Razzari, F. Légaré, R. Morandotti, S. Chu, B. E. Little, and D. J. Moss, Opt. Express 18, 923 (2010).

7. M. Ferrera, D. Duchesne, L. Razzari, M. Peccianti, R. Morandotti, P. Cheben, S. Janz, D.-X. Xu, B. E. Little, S. Chu, and D. J. Moss, Opt. Express 17, 14098 (2009).

8. J. Hu, X. Sun, A. Agarwal, and L. C. Kimerling, J. Opt. Soc. Am. B 26, 1032 (2009).

9. Q. Xu, D. Fattal, and R. G. Beausoleil, Opt. Express 16, 4309 (2008).

10. Q. Xu, B. Schmidt, S. Pradhan, and M. Lipson, Nature 435, 325 (2005).

11. P. Dong, S. Liao, D. Feng, H. Liang, D. Zheng, R. Shafiiha, C.-C. Kung, W. Qian, G. Li, X. Zheng, A. V. Krishnamoorthy, and M. Asghari, Opt. Express 17, 22484 (2009).

12. A. Biberman and K. Bergman, Rep. Prog. Phys. 75, 046402 (2012).

13. C. Qiu, J. Shu, Z. Li, X. Zhang, and Q. Xu, Opt. Express 19, 5143 (2011).

14. R. A. Soref and B. R. Bennett, IEEE J. Quantum Electron. 23, 123 (1987). 